Nora Ketschik, Benjamin Krautter, Sandra Murr und Yvonne Zimmermann

\title{
Zur Theorie von Erzählebenen und ihrer Annotation im digitalen Kontext
}

Zusammenfassung: Der vorliegende Beitrag ist im Rahmen eines Shared Task zur Analyse von Erzählebenen durch Annotation (SANTA) entstanden und beschäftigt sich mit der formalen Erfassung des narratologischen Konzepts ,Erzählebene'. Der Beitrag setzt sich zunächst mit der Theorie von Erzählebenen in der Literaturwissenschaft auseinander, leitet daraus Merkmale für die Erkennung von Erzählebenen ab und entwickelt schließlich Richtlinien für ihre Annotation. Eine wesentliche Erkenntnis der definitorischen Arbeit liegt in der Verknüpfung des Konzepts ,Erzählebene' mit der Rolle des Erzählers. Indem unterschiedliche Erzählertypen identifiziert werden, können verschiedene Szenarien für die Entstehung neuer Erzählebenen aufgezeigt und kategorisiert werden. Hierbei werden nicht nur prototypische Fälle behandelt, sondern auch seltene und problematische Fälle berücksichtigt. Unser Ziel besteht darin, sowohl eine reflektierte theoretische Auseinandersetzung mit Erzählebenen zu leisten als auch eine akkurate Erfassung des Phänomens zu ermöglichen. Unser methodisches Vorgehen - die Annäherung an ein Phänomen durch Annotation - hat sich dafür als äußert ertragreich erwiesen.

Abstract: This contribution was written in the context of the Shared Task on the Analysis of Narrative Levels through Annotation (SANTA) and deals with the formalization of the narratological concept of 'narrative level'. Firstly, we discuss the theory of narrative levels in literary studies. Secondly, we derive features for the identification of narrative levels and finally, we develop guidelines for their annotation. An essential finding of the theoretical work lies in connecting the concept of 'narrative level' to the narrator. By identifying different types of narrators, we are able to enumerate and categorize different scenarios for the emergence of new levels in narrative texts. Hereby, the article does not remain restricted to prototyp-

Anmerkung: Bei dem hier vorliegenden Artikel handelt es sich um eine Überarbeitung und Übersetzung der zweiten Version unserer Guidelines, mit denen wir am Shared Task on Analysis of Narrative Levels through Annotation (SANTA) teilgenommen haben. Sie wird derzeit für eine Publikation im Journal Cultural Analytics vorbereitet.

Nora Ketschik, Yvonne Zimmermann, Institut für Literaturwissenschaft, Universität Stuttgart Sandra Murr, Stuttgart Research Center for Text Studies, Universität Stuttgart

Benjamin Krautter, Germanistisches Seminar, Universität Heidelberg

Ә Open Access. (C) 2020 Nora Ketschik, Benjamin Krautter, Sandra Murr und Yvonne Zimmermann; publiziert von De Gruyter (c) BY-NC-ND Dieses Werk ist lizenziert unter der Creative Commons AttributionNonCommercial-NoDerivatives 4.0 International Lizenz. https://doi.org/10.1515/9783110693973-018 
ical examples, but also deals with rare and problematic cases. Overall, our goal is both to provide a theoretical reflection on narrative levels and to create accurate guidelines for its recognition. Our methodological approach, i. e. addressing the phenomenon through annotation, has proven to be extremely fruitful.

\section{Vorbemerkung}

Im Rahmen einer Ausschreibung zur digitalen Annotation von Erzählebenen, die im Frühjahr 2018 unter dem Titel Shared Task on the Analysis of Narrative Levels Through Annotation veröffentlicht wurde, haben wir uns intensiv mit der theoretischen und praktischen Erfassung von Erzählebenen beschäftigt. Die Teilnahme am Shared Task zielte darauf ab, das Konzept der Erzählebene innerhalb von Richtlinien für eine zunächst händische, letztlich aber computergestützte Annotation aufzubereiten. Die Erstellung der Annotationsrichtlinien förderte die Einsicht zutage, dass es sich bei dem narratologischen Konzept ,Erzählebene‘ um ein überaus komplexes Phänomen handelt. Die für die Annotation nötige exakte Bestimmung des Phänomens hat zu Erkenntnissen geführt, die in der üblichen literaturwissenschaftlichen Praxis nur selten bedacht werden. Auf diese Weise haben wir - angeleitet durch die interdisziplinäre Perspektive - neue Einsichten in ein vermeintlich vertrautes Phänomen gewinnen können.

Im Zuge der Ausschreibung haben wir zwei Annotationsrichtlinien erstellt. Die erste Version wurde im Rahmen eines Workshops im September 2018 diskutiert und evaluiert (Ketschik et al. 2019). Im Anschluss an diesen bestand die Möglichkeit, eine revidierte Version der Richtlinien zu erstellen, in der das Feedback aus dem Workshop berücksichtigt werden konnte. In unserem Fall wurden insbesondere literaturwissenschaftliche Begriffe genauer definiert, die Rolle des Erzählers präzisiert und ein möglicher Zusammenhang mit verwandten narratologischen Konzepten diskutiert, vornehmlich der Stellung des Erzählers und Formen der Anachronie.

Wir sind davon überzeugt, dass die Bedeutung des Erzählers und dessen Vermittlungsakt der Schlüssel zu einer adäquaten Beschreibung von Erzählebenen ist. In der Überarbeitung unserer Richtlinien haben wir deshalb nicht nur die Relevanz des Erzählers hervorgehoben, wir haben uns auch dazu entschieden, narratologische Analysen etwa zur Fokalisierung, zum fiktiven Zuhörer des Erzählakts oder zu Parallelphänomenen wie Imaginationen oder Träumen so weit wie möglich auszublenden. Wo notwendig, grenzen wir in den folgenden Ausführungen das Konzept der Erzählebene von anderen Phänomenen ab. Darüber hinaus haben wir die zweite Version unserer Annotationsrichtlinien neu strukturiert.Ziel war es dabei, eindeutiger zwischen unserer theoretischen Konzeption, der Iden- 
tifikation neuer Erzählebenen und der konkreten Annotationsanleitung zu unterscheiden. Um dem Rezipienten das Verständnis unseres theoretischen Ansatzes zu erleichtern, sind ferner zusätzliche Beispiele für verschiedene (Standard- sowie Sonder-)Fälle von Erzählebenen eingearbeitet worden. Der vorliegende Text entspricht in weiten Teilen der zweiten Version unserer Guidelines.

Unser Hauptbestreben besteht darin, das narratologische Konzept von Erzählebenen vollständig zu erfassen und zu formalisieren. In diesem Sinne lehnen wir es ab, das Phänomen zu vereinfachen, nur um ein höheres Maß an intersubjektiver Übereinstimmung zu erreichen (inter-annotator agreement, vgl. dazu den Beitrag von Reiter (2020) ab S. 193 in diesem Band). Stattdessen möchten wir mit den folgenden Ausführungen einen Beitrag zur theoretischen Auseinandersetzung und zu einer möglichst akkuraten Erfassung von Erzählebenen leisten.

\section{Theoretische Einführung und Anwendungsfälle}

Die Narratologie bildet seit den frühen 1960er-Jahren ein zentrales Beschäftigungsfeld von Literaturwissenschaftlerinnen (vgl. Martínez und Scheffel 2009, S. 7). Sie befasst sich mit den Techniken des Erzählens und richtet ihren Fokus auf die systematische Beschreibung unterschiedlicher Typen, Strukturen und Funktionen narrativer Phänomene. Während ihre Begriffe längst als heuristische Instrumente der Textanalyse Anwendung finden, diskutieren Narratologen noch immer, ob die Narratologie eine Methode, eine Theorie oder eine eigenständige Disziplin sei (vgl. Meister 2014, S. 623).

Die Gegenstände der Narratologie umfassen eine große Bandbreite an Phänomenen - man denke an die unterschiedlichen Parameter zur Bestimmung des Erzählers, etwa sein Verhältnis zur erzählten Welt, die von ihm eingenommene Perspektive bzw. Fokalisierung, seine Darstellungsform oder die Zuverlässigkeit seiner Aussagen. Aber auch strukturelle Textelemente, wie der Einsatz von Erzählebenen oder Anachronien, sind Gegenstand narratologischer Analysen. Untersucht werden unter anderem die Ordnung, Dauer und Frequenz des Erzählten. Narratologen widmen sich somit vornehmlich der Struktur und dem Aufbau literarischer Texte. Um sowohl den zeitlichen Handlungsverlauf als auch seine Anordnung und Darstellung in einem literarischen Text zu beschreiben, hat Gérard Genette die Konzepte discours und histoire systematisch voneinander abgegrenzt (vgl. Genette 1994, S. 199-201). Damit trennt er die Frage nach dem ,Was“ der Erzählung von der Frage danach, ,wie‘ etwas erzählt wird. Während die histoire „die Gesamtheit der erzählten Ereignisse“ (Genette 1994, S. 199) subsumiert, meint der 
discours die tatsächliche Umsetzung der histoire im jeweiligen Erzählakt, sei er mündlich oder schriftlich überliefert.

Um Erzählebenen voneinander zu unterscheiden, schlägt Genette eine Klassifizierung verschiedener Erzählertypen vor. Hierfür verwendet er die Begriffe ,extradiegetisch', ,intradiegetisch' und ,metadiegetisch'. Der extradiegetische Erzähler führt in die „primäre Erzählung mitsamt ihrer Diegese“ (Genette 1994, S. 249), sprich ihrer Erzählwelt, ein. In dieser Erzählung kann sich wiederum ein intradiegetischer Erzähler befinden - meist eine Figur der ersten Erzählebene, die innerhalb dieser eine eigenständige Erzählung anstößt. Dieses Schema lässt sich beliebig erweitern. Üblicherweise wird diese Struktur metaphorisch beschrieben, indem etwa von einer Rahmung (framing) oder Verschachtelung (embedding) gesprochen oder das Phänomen mit chinesischen Schachteln, Tablettständern oder Matruschkas verglichen wird (vgl. Ryan 1991, S. 178-182, vgl. Pier 2014, S. 547).

In unseren Annotationsrichtlinien folgen wir Genettes grundlegender Annahme, dass eine Erzählebene eine ausreichend erkennbare „Schwelle zwischen den einzelnen Diegesen“ (Genette 1994, S. 249) benötigt. Um diese Schwelle zu definieren, knüpft Genette die Existenz einer neuen Erzählebene an die Einführung eines neuen Erzählers. Die Erzählebene entsteht also, weil ein neuer, in der fiktiven Welt der Diegesis situierter Sprecher eine Geschichte erzählt, die in einer neuen Welt angesiedelt ist bzw. neue Figuren an einem neuen Ort zu einer anderen Zeit zum Gegenstand hat oder haben kann (vgl. Ryan 1991, S. 175-177). Während dieser Sprecher seine Geschichte erzählt, wird er zu einem intradiegetischen Erzähler. Der Erzählerwechsel ist damit ursächlich für den Wechsel der Erzählebene. Der orientalische Märchenzyklus Tausendundeine Nacht etwa wird schon bei Genette als Beispiel für diese Art des Erzählerwechsels herangezogen.

Grundsätzlich wollen wir an dieser Annahme Genettes festhalten. Da die Literaturgeschichte jedoch Beispiele hervorgebracht hat, die auch ohne prototypischen Erzählerwechsel deutliche Anzeichen einer neuen Erzählebene aufweisen (vgl. Lahn und Meister 2013, S. 83), werden wir sie dennoch erweitern. Solche Fälle gilt es, akkurat zu beschreiben: Sie müssen einerseits eine eindeutig abgrenzbare Erzählwelt aufweisen, sind andererseits aber sowohl von Formen der Anachronie als auch von figuralen Sprechakten eindeutig zu unterscheiden. Zwar können Sprechakte ein Indikator für die Einführung einer neuen Erzählebene sein (vgl. Ryan 1991, S. 175-177), alleine stellen sie hierfür allerdings kein ausreichendes Kriterium dar. Um einen Wechsel der Erzählebene herbeizuführen, muss ein eingeführter Sprecher nach unserem Verständnis eine Geschichte ,erzählen` und damit zum Erzähler werden. Tatsächlich kommt dies in Sprechakten aber eher selten vor.

Die Annotation von Erzählebenen kann ohne ein umfassendes Verständnis erzähltexttheoretischer Theoreme letztlich nicht gelingen. Dieses ist notwendig, 
um die Struktur eines Textes zu analysieren und damit Einsichten in das Verhältnis von Form und Inhalt zu erhalten. ${ }^{1}$ Dass für die Analyse von Erzähltexten die Erfassung von Erzählebenen von hoher Relevanz ist, erschließt sich letztlich auch daraus, dass diese den Aufbau und Inhalt des Texts ebenso wie den Einsatz unterschiedlicher Erzähler aufschlüsseln.

Mögliche Forschungsfragen, die sich an die Analyse von Erzählebenen anschließen, konzentrieren sich vorwiegend auf die strukturelle Untersuchung eines Texts. Man denke etwa an die Unterscheidung der vorkommenden Erzähler, das Verhältnis von Rahmen- und Binnenerzählung oder die Bedeutung der einzelnen Erzählungen für das Gesamtgefüge. Forschungsfragen können sich aber natürlich auch dem Inhalt der Erzählung(en) widmen. Da die Erzählebenen funktional aufeinander bezogen werden können, ist es wichtig, die Informationsverteilung der Figur/en oder der/s Erzähler/s unter Berücksichtigung solcher Interdependenzen zu interpretieren. Erzählebenen können im Textganzen unterschiedliche Funktionen einnehmen. Von einer explikativen Funktion wird gesprochen, wenn die eingeschobene Erzählung Erklärungen für Elemente der Rahmenerzählung bereitstellt. Eine aktionale Funktion hingegen liegt vor, ist die eingebettete Erzählung für die Haupterzählung handlungskonstitutiv. Und eine thematische Funktion findet sich, wenn es zwischen den beiden Erzählungen auf struktureller Ebene Analogien, Korrespondenzen oder Kontrastbeziehungen gibt (vgl. Lahn und Meister 2013, S. 83 f.). Darüber hinaus scheint uns eine systematischere Beschreibung der Übergänge von einer Erzählebene zur anderen für das Erkennen solcher Phänomene instruktiv zu sein. Die detaillierte Erfassung hilft, das Phänomen zu verstehen und damit die Ebenen besser identifizieren zu können. Mit Blick auf die Literaturgeschichte als Ganzes wäre es ebenfalls aufschlussreich, genauer zu untersuchen, ob sich für Anzahl, Länge und Funktion von Erzählebenen bestimmte Muster hinsichtlich literarischer Gattungen, literarischer Perioden, des Geschlechts oder der Herkunft des Autors ablesen lassen.

\section{Narratologische Begriffe und Konzepte}

Im Folgenden wollen wir einige grundlegende narratologische Begriffe, die wir in unseren Grundannahmen (Abschnitt 4) und den Annotationsrichtlinien (Abschnitt 5) verwenden, kursorisch erläutern. Dies soll zu einem besseren Verständnis unserer Richtlinien und der ihnen zugrundeliegenden literaturwissenschaftlichen Konzeption beitragen.

1 Vgl. etwa das ,Gehalt-Gestalt-Gefüge‘, dazu kursorisch Klausnitzer 2013, S. 126 f. 


\subsection{Erzählung}

Obwohl der Begriff der Narrativität inzwischen als umstritten gilt (vgl. Abbot 2014, S. 587), werden in der Literaturwissenschaft immer noch zwei unterschiedliche Konzepte verwendet: Während Narrativität in der klassischen Erzähltheorie „an die Gegenwart einer vermittelnden Instanz, des ,Erzählers‘, gebunden“ ist (Schmid 2014, S. 1), fokussiert der Strukturalismus vor allem die „temporale Struktur und [...] Veränderungen eines Zustands“(Schmid 2014, S. 2). Wir stimmen mit Wolf Schmid überein, dass Narrativität in „practical literary theory“ am besten durch eine Kombination beider Konzepte erklärt werden kann (Schmid 2003, S. 17). Die Erzählung ist somit kommunikationstheoretisch betrachtet mit einem Sprechakt verbunden, da sie Teil eines Vermittlungsaktes ist. Der Erzähler adressiert seine Geschichte stets an einen fiktiven Zuhörer, egal ob dieser explizit erwähnt wird oder nicht. Unsere Minimaldefinition einer in einem Erzählakt präsentierten Geschichte ${ }^{2}$ lautet, dass eine Handlung einen Zustandswechsel von einem Zustand $A$ zu einem Zustand $B$ umfasst, dass dieser Zustandswechsel motiviert und kausal verknüpft ist und dass er von einem Erzähler vermittelt wird (vgl. Martínez und Scheffel 2009, S. 109-110 und Schmid 2018, S. 312 f.).

\subsection{Der Erzähler}

Die Analyse des Erzählers gehört zu den zentralen Aufgaben der Narratologie (vgl. Igl 2018, S. 127). ${ }^{3}$ Um unterschiedliche Erzähler voneinander abgrenzen und damit auch den Wechsel des Erzählers identifizieren zu können, ist es sinnvoll, die Stellung des Erzählers in Bezug auf die von ihm erzählte Geschichte zu bestimmen. Daher ist grundsätzlich zu klären, ob er Teil der von ihm erzählten Welt ist oder nicht (vgl. Genette 1994, S. 174-176).

2 Genettes Begriff histoire wird im Deutschen als ,Geschichte‘ übersetzt. Wir meinen damit nicht nur den gesamten Text, sondern auch die Geschichten einzelner Erzählebenen.

3 Tatsächlich gibt es in der Narratologie unterschiedliche Positionen hinsichtlich der Bedeutung des Erzählers: Es wird immer noch diskutiert, ob der Erzähler „the highest-level speech position“ in einem literarischen Text innehat und damit eine „strictly textual category“ ist, die deutlich vom Autor zu unterscheiden sein sollte (vgl. Margolin 2014, S. 646), oder ob narrative Texte ohne Erzähler denkbar sind (vgl. Igl 2018, S. 128 f.). 


\subsubsection{Der homodiegetische Erzähler}

Ein homodiegetischer Erzähler ist als Figur in der von ihm erzählten Welt Teil seiner eigenen Geschichte: sei es als stiller Beobachter, Nebenfigur oder Hauptfigur. ${ }^{4}$ In retrospektiv erzählten Geschichten spaltet sich somit das Ich des homodiegetischen Erzählers in ein ,erzählendes` und ein ,erlebendes Ich` auf. Während das erzählende Ich auf der primären Erzählebene in der Exegesis, ansonsten aber auf einer übergeordneten Erzählebene situiert ist und von dieser Warte aus einen Überblick über den gesamten Handlungsverlauf hat, sind die Gedanken und Gefühle des erlebenden Ichs an die aktuelle Handlungssituation gebunden und vom gerade Erlebten geprägt (vgl. Lahn und Meister 2013, S. 70; Fludernik 2006, S. $103 \mathrm{f}$.). Normalerweise ist eine klare Trennung von erzählendem und erlebendem Ich wahrzunehmen, da ein Unterschied existiert zwischen dem Zeitpunkt, zu dem erzählt wird, und dem Moment, in dem erlebt wird. Es finden sich aber auch Texte, in denen die Distanz zwischen den beiden Ichs scheinbar aufgehoben wird (vgl. etwa im inneren Monolog in Arthur Schnitzlers Leutnant Gustl, Lahn und Meister 2013, S. 72).

\subsubsection{Der heterodiegetische Erzähler}

Im Gegensatz zum homodiegetischen Erzähler ist der heterodiegetische Erzähler nicht Teil der von ihm erzählten Welt. In einer Geschichte, die von einem heterodiegetischen Erzähler vermittelt wird, gibt es daher stets eine klare Unterscheidung zwischen dem Standpunkt des Erzählers in der Exegesis und der Welt der Figuren in der Diegesis. Da er nicht Teil der erzählten Welt ist, gibt es hier auch keine Aufspaltung des Erzählers in ein erzählendes und erlebendes Ich.

\subsection{Erzählebenen}

Um das Phänomen von Erzählebenen zu beschreiben, haben sich in der Literaturwissenschaft viele teils sehr unterschiedliche Begrifflichkeiten ausgebildet (vgl. Pier 2014, S. 549-558). Wir fokussieren uns darauf, jede neue Erzählung eines Erzähltexts zu identifizieren und sie als neue Erzählebene zu definieren. Eine neue Erzählung wird nach unserem Verständnis von einem Erzählerwechsel ausgelöst.

4 Der Begriff des autodiegetischen Erzählers hat sich für einen homodiegetischen Erzähler durchgesetzt, der die Hauptfigur seiner Geschichte ist. Für unsere Analysen ist diese Unterscheidung nicht relevant. 


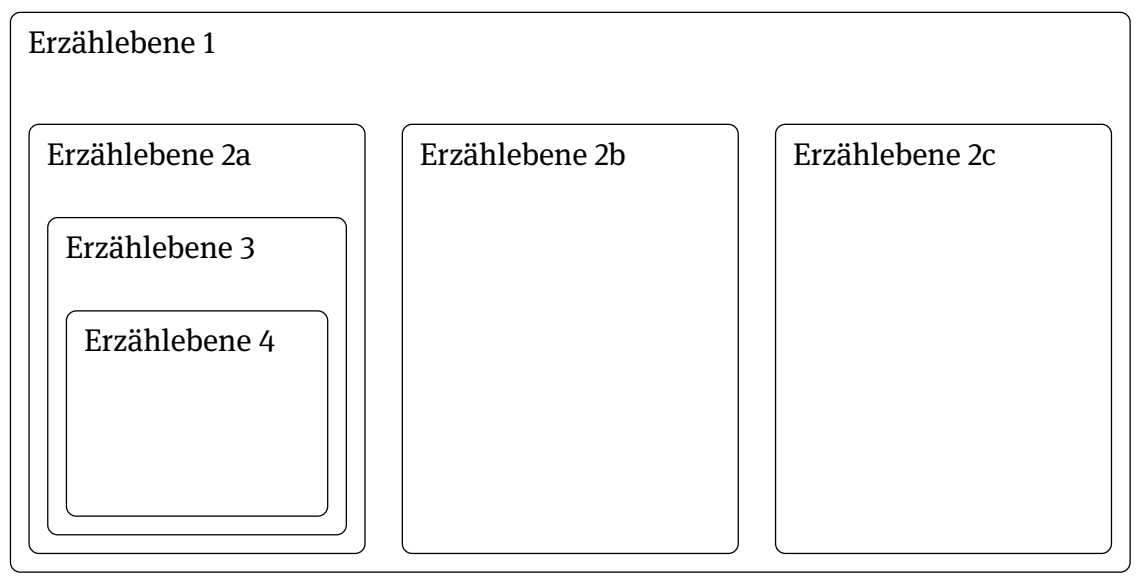

Abb. 1: Ineinander verschachtelte und sequenziell angeordnete Erzählebenen in einem literarischen Text

In den meisten Fällen ist dieser Wechsel eindeutig ersichtlich. Es gibt allerdings durchaus Erzählerwechsel, die subtiler angelegt und daher schwieriger zu greifen sind (dies wird in Abschnitt 4.2 genauer beschrieben).

Erzählebenen können ineinander verschachtelt (s. Abbildung 1: Ebenen 1 bis 4) oder sequenziell verknüpft sein (ebd.: 2a, 2b, 2c) oder aber eine Kombination aus beidem darstellen. In eine Rahmenerzählung bzw. eine übergeordnete Erzählebene können mehrere Erzählungen eingebettet sein. Da eine eingebettete Erzählung wiederum selbst für eine andere eingebettete Erzählung zur Rahmenerzählung werden kann, sprechen wir in unseren Guidelines von primären, sekundären, tertiären etc. Erzählebenen. Dadurch wird auch die Ambiguität von Genettes Terminologie (extra-, intra-, meta-, metametadiegetisch etc.) vermieden (vgl. Jahn 2017). Werden Erzählebenen sequenziell angeordnet, befinden sie sich grundsätzlich auf derselben Ebene. Sequenzielle Erzählebenen sind normalerweise immer schon auf einer zweiten, dritten, vierten etc. Ebene angeordnet und werden nur sehr selten ohne Rahmung auf der ersten Ebene aneinandergereiht (ein Beispiel hierfür wäre etwa Orhan Pamuks Rot ist mein Name).

\subsection{Exegesis und Diegesis}

„[D]iegesis designates the level of the narrated world, and exegesis the level of the narrating“, konstatieren Coste und Pier (2009, S. 301). Dementsprechend gehört ein homodiegetischer Erzähler der ersten Erzählebene immer schon zu beiden Be- 
reichen: In seiner Funktion als Erzähler gehört er der Exegesis an; da er aber eine Geschichte erzählt, an der er selbst teilhat, agiert er auch auf der Ebene der Diegesis (vgl. Genette 1994, S. 249). Ein heterodiegetischer Erzähler hingegen ist nur in der Exegesis situiert, da er nicht Teil der erzählten Welt ist.

Die Exegesis, also der Standort des Erzählers in einer primären Erzählsituation, bildet keine eigenständige Erzählebene. In diesem Sinne verweisen Aphorismen, Motti, Kommentare, Wertungen und Adressatenanreden ${ }^{5}$ nicht auf eine eigenständige Erzählebene (vgl. Schmid 2014, S. 7), sondern sind Teil der aktuellen Erzählebene. Dies gilt gleichfalls für Aussagen von sekundären, tertiären etc. Erzählern, soweit sie nicht Elemente der übergeordneten Erzählebene adressieren (s. Abschnitt 5.8).

\subsection{Illokutionäre und ontologische Grenzen}

In ihrer Untersuchung von Erzählebenen schlägt Marie-Laure Ryan vor, sich auf den Anfang und das Ende von Erzählsträngen zu konzentrieren. Dabei unterscheidet sie illokutionäre von ontologischen Grenzen, die beide sowohl tatsächlich als auch virtuell überschritten werden können (vgl. Ryan 1991, S. 175-177). ${ }^{6}$ Nach unserem Verständnis reicht allerdings weder ein Sprecherwechsel, also die Überschreitung der illokutionären Grenze, noch die Präsentation eines Sprechakts durch den Erzähler allein aus, um eine neue Erzählebene zu begründen. Es handelt sich bei diesen Fällen zuallererst um Sprechakte, die lediglich unter bestimmten Bedingungen (vgl. hierzu Abschnitte 4.1 und 4.2) zu einem Erzählerwechsel führen können und damit eine neue Erzählebene einleiten. Ontologische Grenzen führen nur in ganz bestimmten Fällen zu neuen Erzählebenen, nämlich

5 Im fiktionalen Text sind damit stets Anreden des fiktiven Zuhörers bzw. Lesers gemeint.

6 Ryan schreibt: „Illocutionary boundaries delimit speech acts within a text or a conversation, and their crossing introduces a new speaker or a new narrator" (Ryan 1991, S. 175). Dabei unterscheidet sie vier Formen der Grenzüberschreitung, zwei Formen der illokutionären und zwei Formen der ontologischen Grenzüberschreitung. Eine tatsächlich überschrittene illokutionäre Grenze werde durch einen neuen Sprecher eingeführt, was sich auf der Mikroebene durch zitierte Figurenrede und auf der Makroebene zum Beispiel durch „narratives of personal experience“ zeige (Ryan 1991, S. 176). Virtuell überschrittene illokutionäre Grenzen hingegen werden durch den Erzähler eingeleitet, der den Sprechakt der Figur etwa in einer indirekten Rede vermittelt (vgl. Ryan 1991, S. 177). Eine ontologische Grenze „transports the reader to a new system of reality“ (Ryan 1991, S. 177). Wird die Grenze tatsächlich überschritten, wird das neue System als Ort der fiktionalen Realität beschrieben - zumindest vorübergehend. Das neue Realitätssystem wird also aus seinen eigenen Grenzen heraus bestimmt. Wird die Grenze hingegen virtuell überschritten, wird das neue System an einer „external perspective“ verankert, wobei das System „with repeated reminders of its [...] status in the primary reality“ verstanden wird (Ryan 1991). 
dann, wenn mit ihnen ein neuer Erzähler eingeführt wird. Deshalb eröffnet beispielsweise Harry Potters Sprung durch die Mauer am Bahnhof King's Cross nach unserem Verständnis auch keine neue Erzählebene.

\section{Grundannahmen: Wie findet man Erzählebenen?}

Mit den folgenden Grundannahmen lassen sich alle Erzählebenen eines literarischen Textes auffinden. Generell gehen wir davon aus, dass in jedem Text mindestens eine Erzählebene vorliegt.

\subsection{Neue Erzählebene durch neue Geschichte}

Eine neue Erzählebene liegt vor, wenn eine neue Geschichte erzählt wird, die in einem neuen Erzählakt präsentiert wird. Sie wird durch einen Erzählerwechsel kenntlich gemacht. Den Begriff des Erzählerwechsels nutzen wir in einem weiten Sinn, der auch die Funktion des Erzählers miteinschließt. Deshalb liegt auch dann ein Erzählerwechsel vor, wenn sich die Stellung des Erzählers zum Erzählten ändert (s. Abschnitt 3.2). Eine Geschichte zeichnet sich prinzipiell durch folgende Kriterien aus:

1. Eine Geschichte ist eine in sich geschlossene Handlung, deren Ereignisse und Geschehnisse kausal miteinander verbunden sind und eine Zustandsveränderung bewirken.

2. Geschichten werden durch einen Erzähler vermittelt und als der „mündliche[] oder schriftliche[] Diskurs“ präsentiert, „der von einem Ereignis oder einer Reihe von Ereignissen“ berichtet (Genette 1994, S. 17).

3. Das Tempus von Erzählungen ist vornehmlich das Präteritum. In neuer Zeit gibt es einige Ausnahmen, etwa die Erzählung Faserland von Christian Kracht, in der ein homodiegetischer Erzähler im Präsens erzählt.

\subsection{Fälle von Erzählerwechsel}

Ein Erzählerwechsel führt immer zu einer neuen Geschichte, womit zugleich eine neue Erzählebene beginnt. Die Komplexität literarischer Texte zwingt uns allerdings, Genettes Annahmen vom Erzählerwechsel auszuweiten. Im Folgenden erläutern wir konkrete Szenarien, die einen Erzählwechsel und den Beginn einer neuen Erzählebene einleiten: 


\subsubsection{Der prototypische Fall: eine neue Erzählfigur}

Üblicherweise ergibt sich ein Erzählerwechsel dadurch, dass eine Figur der erzählten Welt eine, wenn nicht gar seine eigene Geschichte erzählt. Damit ist der Erzähler zweiter Stufe Teil der Diegesis, die vom übergeordneten Erzähler vermittelt wird. In einer neuen Erzählung dieser Art findet sich somit ein neues erzählendes Ich.

Diese Variante entspricht Genettes Verständnis von Erzählebenen (vgl. Genette 1994, S. 250). Sein Beispiel aus dem von Abbé Prévost verfassten Roman Histoire du Chevalier Des Grieux et de Manon Lescaut (dt. Manon Lescaut) verweist auf den prototypischen Erzählerwechsel (vgl. Genette 1994, S. 162-164). Der Roman beginnt mit den Memoiren des Monsieur de Renoncour. Er ist der primäre Erzähler, bei dem es sich - da er seine eigene Geschichte erzählt - um einen homodiegetischen Erzähler handelt. Der Roman setzt wie folgt ein:

Ich muss meine Leser in die Zeit meines Lebens zurückversetzen, zu der ich dem Chevalier des Grieux zum ersten Mal begegnet bin. Es war ungefähr ein halbes Jahr vor meiner Abreise nach Spanien. Obschon ich meine Abgeschiedenheit nur selten verließ, unternahm ich doch meiner Tochter zuliebe ab und zu eine kleine Reise, die ich aber tunlichst abkürzte. (Abbé Prévost 2008, S. 5)

Die in einer Analepse erläuterte Erklärung de Renoncours, die darlegt, wie er einst des Grieux traf, nimmt mehrere Seiten ein. Daran anschließend werden zwei Jahre der erzählten Zeit übersprungen. Eines Tages treffen sich de Renoncour und des Grieux in Calais wieder, wo des Grieux von seiner kürzlich begangenen Reise nach Amerika, seinen Unglücksfällen und seinem Versagen berichtet. De Renoncour führt diese Geschichte folgendermaßen ein:

Ich muss meine Leser hier darauf aufmerksam machen, dass ich seine Geschichte fast unmittelbar nachher niedergeschrieben habe, als ich sie von ihm vernommen hatte. Ich kann also versichern, dass dieser Bericht ganz genau und fast wortgetreu ist. Ich meine wortgetreu in der Wiedergabe der Gedanken und Gefühle, die der junge Abenteurer mit dem edelsten Anstand zum Ausdruck brachte. Im Folgenden werde ich demnach seinem Bericht gar nichts hinzufügen, was nicht von ihm selbst stammt.

Ich war siebzehn Jahre alt und vollendete meine Philosophiestudien in Amiens, wo meine Eltern, die einem der vornehmsten Häuser von P... angehörten, mich hingeschickt hatten. Ich führte ein so geordnetes, sittsames Leben, dass meine Lehrer mich der ganzen Schule als Vorbild hinstellten. Ich gab mir zwar nicht sonderlich Mühe, dieses Lob zu verdienen, aber ich bin von Natur sanft und ruhig veranlagt. Ich lag aus Neigung meinen Studien mit großem Eifer ob, und man rechnete mir eine gewisse Abneigung gegen Ausschweifungen als Tugend an. Meine Herkunft, meine erfolgreichen Studien und einige äußere Vorzüge, über die ich verfügte, hatten mir die Bekanntschaft und die Wertschätzung aller achtbaren Leute in der Stadt verschafft. (Abbé Prévost 2008, S. 13) 
Als eine Figur auf der ersten Erzählebene wird aus des Grieux ein intradiegetischer Erzähler bzw. nach unserer Terminologie ein sekundärer Erzähler. Sein Sprechakt wird zu einem Erzählakt, womit ein neues erzählendes Ich entsteht. Die direkte Rede in der ersten Erzählebene indiziert einen Sprecherwechsel. Erst indem des Grieux seine Geschichte erzählt, die sich durch in sich geschlossene Handlungen auszeichnet, deren Ereignisse und Geschehnisse kausal miteinander verbunden sind und die eine Zustandsänderung bewirken, wird er vom einfachen Sprecher zum wirklichen Erzähler. Irrelevant bleibt dabei, dass seine Geschichte gleichzeitig eine ontologische Grenze überschreitet, eben weil die Reise nach Amerika eine Welt beschreibt, die sich von der Welt de Renoncours unterscheidet.

In der Literaturgeschichte kommen ähnliche Textstrukturen recht häufig vor. Heinrich von Kleists Erzählung Unwahrscheinliche Wahrhaftigkeiten ist eines von vielen weiteren Beispielen, das an dieser Stelle angeführt werden kann. In Kleists Anekdote liegt auf der ersten Erzählebene ein heterodiegetischer Erzähler vor. Er beginnt seine Geschichte wie folgt:

„Drei Geschichten“, sagte ein alter Offizier in einer Gesellschaft, „sind von der Art, daß ich ihnen zwar selbst vollkommen Glauben beimesse, gleichwohl aber Gefahr liefe, für einen Windbeutel gehalten zu werden, wenn ich sie erzählen wollte. Denn die Leute fordern, als erste Bedingung, von der Wahrheit, daß sie wahrscheinlich sei; und doch ist die Wahrscheinlichkeit, wie die Erfahrung lehrt, nicht immer auf Seiten der Wahrheit.“

Erzählen Sie, riefen einige Mitglieder, erzählen Sie! - denn man kannte den Offizier als einen heitern und schätzenswürdigen Mann, der sich der Lüge niemals schuldig machte.

Der Offizier sagte lachend, er wolle der Gesellschaft den Gefallen tun; erklärte aber noch einmal im Voraus, daß er auf den Glauben derselben, in diesem besonderen Fall, keinen Anspruch mache.

Die Gesellschaft dagegen sagte ihm denselben im Voraus zu; sie forderte ihn nur auf, zu reden, und horchte.

„Auf einem Marsch 1792 in der Rheinkampagne“, begann der Offizier, „bemerkte ich, nach einem Gefecht, das wir mit dem Feinde gehabt hatten, einen Soldaten, der stramm, mit Gewehr und Gepäck, in Reih' und Glied ging, obschon er einen Schuß mitten durch die Brust hatte; wenigstens sah man das Loch vorn im Riemen der Patrontasche, wo die Kugel eingeschlagen hatte, und hinten ein anderes im Rock, wo sie wieder herausgegangen war [...].“ (Kleist 1990, S. 376)

Der Erzählerwechsel ist deutlich erkennbar, wenn der Offizier mit den Worten „Auf einem Marsch 1792 in der Rheinkampagne“ einsetzt. Hier beginnt der Offizier als homodiegetischer Erzähler, eine Geschichte zu erzählen, die er selbst erlebt hat. Mit Ausnahme des verbum dicendi „begann der Offizier“ gehört die folgende Passage zur zweiten Erzählebene. 


\subsubsection{Wechsel der Erzählposition}

Obgleich die durch eine erzählende Figur eingeläutete Binnenerzählung der Standardfall eines Erzählebenenwechsels ist, gibt es literarische Texte, die von diesem prototypischen Szenario abweichen. In selteneren Fällen kann ein Erzählerwechsel vorliegen, ohne dass die Erzählfigur wechselt. So etwa, wenn ein extradiegetisch-homodiegetischer Erzähler plötzlich eine Geschichte erzählt, an der er entweder nicht teilhat oder die in seiner erzählten Welt selbst fiktional ist. In diesem Fall bleibt das erzählende Ich zwar dasselbe, seine Stellung zum Erzählten ändert sich jedoch.

Wir schlagen vor, auch in diesem Fall von einem Erzählerwechsel zu sprechen und unterscheiden damit zwischen dem Erzähler als Figur und seiner analytischen Funktion als Erzähler. Während ein neuer homodiegetischer Erzähler durch eine erzählende Figur eingeführt wird, ist ein neuer heterodiegetischer Erzähler auf zweiter, dritter etc. Stufe meist durch seine veränderte Stellung zur erzählten Welt auszumachen. Ein Beispiel für den zweitgenannten Fall findet sich in Max Frischs Roman Stiller. James White alias Anatol Ludwig Stiller erscheint über weite Teile des Romans als homodiegetischer Erzähler. Während seiner Gefangenschaft erzählt er seinem Verteidiger das Märchen von ,Rip van Winkle‘:

Dazu (was wichtig ist) hielt ich sein silbernes Feuerzeug mit Flämmchen, ohne jedoch die duftende Zigarre, diese immerhin einzige Wollust in meiner Untersuchungshaft, anzuzünden, nein, aller Begierde zum Trotz wiederholte ich meine Frage:

„Sie kennen es nicht?“

„Was?“

„Das Märchen von Rip van Winkle?“

Nur mit diesem Kniff, nämlich mit dem Feuerzeug in der Hand, das ich nach jedem Verlöschen wieder entzündete, dazu mit der Zigarre in der andern Hand, unablässig im Begriff, die schöne Zigarre endlich anzustecken, ja, einmal schon mit der ersten Glut an der Zigarre, so daß ich bloß hätte ziehen müssen, im letzten Augenblick doch jedesmal wieder verhindert - durch Rip van Winkle, dessen Märchen offensichtlich sogar akuter war als meine Zigarre - nur so konnte ich meinen geschäftigen Verteidiger überhaupt zum Zuhören, zum aufmerksamen Zuhören nötigen.

Das Märchen lautet etwa folgendermaßen:

Rip van Winkle, ein Nachkomme jener unerschrockenen van Winkles, die unter Hendrik Hudson dereinst das amerikanische Land erschlossen hatten, war ein geborener Faulenzer, dabei, wie es scheint, ein herzensguter Kerl, der nicht um der Fische willen fischte, sondern um zu träumen, denn sein Kopf war voll sogenannter Gedanken, die mit seiner Wirklichkeit wenig zu tun hatten. (Frisch 1954, S. 70 f.)

Der Abschnitt verdeutlicht, wie Stiller seine Erzählposition verändert: Nachdem er zu Beginn als homodiegetischer Erzähler auftritt, vermittelt er die Geschichte von Rip van Winkle nun als heterodiegetischer Erzähler. Sobald er beginnt, 
das Märchen zu erzählen, verändert sich seine Stellung zur erzählten Welt. Somit liegt hier ein Wechsel der Erzählebene vor, ohne dass ein neuer Sprecher bzw. eine neue erzählende Figur eingeführt wird. Daraus folgt, dass ein Wechsel von einer homodiegetischen zu einer heterodiegetischen Erzählposition - ohne Wechsel der Sprecherposition - ausreicht, um eine neue Erzählebene zu bilden.

\subsubsection{Transferierende Erzähler: Chronisten, Sammler, Herausgeber}

Manche Erzähler signalisieren eine distanzierte Haltung zu der von ihnen erzählten Geschichte, weil sie die Geschichte explizit als überliefert bezeichnen. Für diese Formen führen wir den Oberbegriff, transferierende Erzähler‘ ein. Häufig äußert ein solcher transferierender Erzähler, dass er zufällig Zugang zu einer Geschichte erhalten hat, etwa indem er ein Manuskript gefunden oder eine Chronik gelesen hat. Für die Gesamterzählung hat dieser Erzähler eine explizit authentifizierende Funktion.

1. Herausgeberfiktion. Als ein Sonderfall narrativer Texte gilt die Herausgeberfiktion: ,an agent whose sole involvement with the text is its material dissemination“ (Ryan 2001, S. 151). Die Herausgeberfunktion bedingt immer eine eigene Erzählebene, auch wenn diese Erzählebene nur aus einem einzigen Satz besteht. Nicht selten kommt die Herausgeberfiktion erst am Ende eines Textes zum Vorschein. Obgleich bis zu diesem Zeitpunkt keine Indizien auf eine Rahmenhandlung hinweisen, macht die Existenz des Herausgebers den bisherigen Text als zweite Erzählebene bzw. Binnenerzählung kenntlich. Aus diesem Grund kann das Inklusionsschema (s. Abschnitt 3.3) grundsätzlich erst erstellt werden, wenn der komplette Text bekannt ist.

Eduard Mörikes Erzählung Lucie Gelmeroth ist ein gutes Beispiel für die Herausgeberfiktion. Der Text setzt mit einem homodiegetischen Erzähler ein, der von seiner Urlaubsreise nach Göttingen erzählt.

Ich wollte - so erzählt ein deutscher Gelehrter in seinen noch ungedruckten Denkwürdigkeiten - als Göttinger Student auf einer Ferienreise auch meine Geburtsstadt einmal wieder besuchen, die ich seit lange nicht gesehen hatte. (Mörike 2005b, S. 13)

Obwohl der erste Satz bereits auf die Präsenz einer Herausgeberfiktion hinweist, indem die „ungedruckten Denkwürdigkeiten“ erwähnt werden, klärt erst das Ende der Erzählung die Verschachtelung der Erzählebenen gänzlich auf. Nach Abschluss der Erzählung ist dort zu lesen:

Hier bricht die Handschrift des Erzählers ab. Wir haben vergeblich unter seinen Papieren gesucht, vom Schicksal jenes flüchtigen Kaufmanns noch Etwas zu erfahren. Auch 
mit Erkundigungen anderwärts sind wir nicht glücklicher gewesen. (Mörike 2005b, S. 29)

Indem von gefundenen „Papieren“ berichtet wird, zeigt sich, dass der Göttinger Student im Grunde ein sekundärer Erzähler ist. Der primäre Erzähler dagegen ist der erst am Ende sichtbar werdende Herausgeber dieser Blätter.

2. Der Erzähler als Berichterstatter. Im Gegensatz zu Herausgebern, die einen Erzähler einführen, der seine Geschichte selbst vermittelt, gibt es Formen der Berichterstattung, in denen ein Erzähler eine aufgefundene Geschichte erzählt. Meist handelt es sich um sogenannte Chronisten. ${ }^{7}$ In Eduard Mörikes Das Stuttgarter Hutzelmännlein erzählt der etwa 1820 positionierte Erzähler die im Jahr 1220 situierte Geschichte von Seppe und Vrone und gibt sich dabei als Chronist zu erkennen. Während Seppe sich in dieser Erzählung mit einem Kutscher unterhält, berichtet der Erzähler von der „Historie der schönen Lau“, die er etwa 100 Jahre vor der Seppe-Erzählung einordnet.

Du aber, wohlgeneigter Leser, lasse dich, derweil die Beiden so zusammen discuriren, auch etlicher Dinge besonders berichten, die, ob sie sich zwar lang vor Seppes Zeit begeben, nichts desto minder zu dieser Geschichte gehören. Vernimm hiernach die wahre und anmuthige

Historie von der schönen Lau.

Der Blautopf ist der große runde Kessel eines wundersamen Quells bei einer jähen Felswand gleich hinter dem Kloster [...]. (Mörike 2005a, S. 130)

In diesem Fall wird weder ein neuer Erzähler eingeführt, noch ändert der Erzähler seine Stellung zur erzählten Welt. Er bleibt heterodiegetisch. Ausmachen lässt sich hingegen etwas anderes: Der Erzähler selbst weist explizit darauf hin, dass eine neue Geschichte, hier Historie, erzählt wird. Diese Geschichte ist nicht mehr als Bestandteil der übergeordneten Erzählung zu betrachten, die von Seppe und Vrone handelt. Vielmehr wird sie als Wissen einer alten Chronik ausgegeben, zu der allein der Erzähler Zugang hat. Die Vermittlung dieses Chronikwissens eröffnet eine neue Erzählebene.

Ähnliche Fälle liegen vor, wenn aus Zeitungsberichten, Briefen, Fernsehsendungen oder ähnlichen Medien Inhalte vermittelt werden, wobei stets eine Vermittlung einer Geschichte vorliegen muss (s. Abschnitt 3.1), um diese als neue Erzählebene auszuzeichnen.

7 Ein Chronist muss, „wie jeder gute Historiker, zumindest die Zuverlässigkeit seiner Quellen und Gewährsleute nachweisen“ (Genette 1994, S. 279). Bei diesen Erzählern ist es nicht immer leicht zu entscheiden, ob es sich um homodiegetische oder heterodiegetische Erzähler handelt (vgl. Genette 1994, S. 262.). Obwohl sie Teil der diegetischen Welt sind, gibt es eine zeitlich unüberwindbare Distanz zwischen ihnen und der erzählten Welt. 


\subsection{Abgrenzung von verwandten Phänomenen}

\subsubsection{Anachronien}

Für ein präzises Verständnis von Erzählebenen ist es wichtig, sie von Anachronien, sprich Analepsen und Prolepsen, zu unterscheiden. Während eine Erzählebene durch einen Erzählerwechsel - oder den Wechsel der Erzählposition - initiiert wird, bleiben Erzähler und Erzählposition in Anachronien konstant. Sie rücken ausschließlich die chronologische Ordnung des Erzählten im discours in den Fokus der Analyse, weshalb anachronistische Passagen die jeweilige Erzählebene nicht verlassen.

Her father was becoming old lately, she noticed; he would miss her. Sometimes he could be very nice. Not long before, when she had been laid up for a day, he had read her out a ghost story and made toast for her at the fire. Another day, when their mother was alive, they had all gone for a picnic to the Hill of Howth. [...]

Her time was running out but she continued to sit by the window, leaning her head against the window curtain, inhaling the odour of dusty cretonne. (Joyce 1967, S. 41)

Der heterodiegetische Erzähler in James Joyces Eveline erzählt die Geschichte von Eveline kurz vor ihrer geplanten Auswanderung. Der Erzähler nutzt mehrere Analepsen, um die Hauptfigur über ihre Kindheit nachdenken zu lassen. Im abgedruckten Abschnitt erinnert sie sich an eine Geistergeschichte ihres Vaters sowie an ein gemeinsames Familienpicknick. Die chronologisch früher erlebten Erinnerungen grenzen sich eindeutig durch ihr Tempus ab. Da aber weder ein Erzählerwechsel vorliegt noch der Erzähler seine Stellung zur erzählten Welt verändert oder gar als transferierender Vermittler auftritt, liegt kein Wechsel der Erzählebene vor.

\subsubsection{Formen der Imagination}

Bestimmte Erzählstrategien setzen figurale Gedanken, Träume, Visionen, Fantasien u. a. so ein, dass ein Wechsel der Erzählebene plausibel erscheinen mag (vgl. Pier 2014, S. 550). In diesen Fällen ist es wichtig, zwischen einem wirklichen Erzählebenenwechsel und einer starken Form interner Fokalisierung zu unterscheiden. In Anton Tschechows Das Gewinnlos stellt sich Ivan Dmitrich, eine der beiden Hauptfiguren, vor, wie das Leben sein könnte, wenn er in der Lotterie gewinnen würde:

Iwan Dmitritsch malt sich den Herbst aus mit seinem endlosen Regen, den kalten Abenden, dem Altweibersommer. In dieser Jahreszeit ist es angenehm, ausgedehnte Spaziergänge zu 
machen im Garten, auf dem Felde, am Ufer des Flusses, um ordentlich durchzufrieren, dann ein großes Gläschen Wodka zu genehmigen und schnell einen eingemachten Reizker nachzuessen oder eine Dillgurke und ... ein zweites Gläschen! Die Kinder kommen aus dem Gemüsegarten gelaufen und schleppen Mohrrüben und Rettich herbei, die noch ganz nach frischer Erde duften ... Dann streckt man sich auf den Diwan, betrachtet in aller Gemütsruhe irgendein illustriertes Journal, breitet zu guter Letzt die Zeitung übers Gesicht, knöpft die Weste auf und verfällt in süßen Schlummer ...(Tschechow 1968, S. 75)

Zwar sind die figuralen Gedanken deutlich erkennbar, allerdings bleiben sie durch den primären Erzähler vermittelt. Wo Ryan - Ivans Vorstellungen als eine neue Welt begreifend - vermutlich für eine ontologische Grenzüberschreitung plädieren würde, reicht dies nach unserem Verständnis nicht für einen Erzählebenenwechsel aus. Denn der heterodiegetische Erzähler vermittelt Ivans Gedanken. Tatsächlich sind seit der Moderne noch radikalere Formen von Gedankenrepräsentationen beobachtet worden, etwa der innere Monolog oder der stream of consciousness (vgl. Martínez und Scheffel 2009, S. 61-63). Erzählformen dieser Art werden vorgeblich nicht mehr durch den übergeordneten Erzähler vermittelt, sondern scheinen direkt von der Figur erlebt und erzählt zu sein (vgl. Igl 2018, S. 127). Prinzipiell ist es vorstellbar, dass auch solche Passagen als neue Ebene des Erzählens gestaltet werden. Die erlebende Figur würde dann jedoch selbst zum Erzähler werden und sich deutlich von der übergeordneten Erzählinstanz unterscheiden.

\subsubsection{Metalepsen}

In einigen wenigen Fällen verwehrt ein Text die Bestimmung von Erzählebenen. Dies ist insbesondere dann der Fall, wenn Erzählebenen erzähllogisch nicht mehr voneinander abgegrenzt werden können, etwa im Falle von Metalepsen (vgl. Pier 2016). In Italo Calvinos Wenn ein Reisender in einer Winternacht ist die Welt des Erzählers bzw. der Leser (die Exegesis) so eng mit der erzählten Welt (der Diegesis) verwoben, dass einzelne Erzählebenen nicht voneinander unterschieden werden können. In solchen Fällen sehen wir von der Annotation jeglicher Erzählebene ab.

Ich bin der Mann, der da zwischen Café und Telefonzelle hin- und herläuft. Oder besser gesagt, dieser Mann heißt hier „ich“, und sonst weißt du nichts von ihm, wie auch dieser Bahnhof nur einfach „Bahnhof“ heißt, und außer ihm gibt es nichts als das unbeantwortete Läuten eines Telefons in einem dunklen Zimmer in einer fernen Stadt. Ich hänge den Hörer ein, warte auf das Scheppern der Münzen durch den metallenen Schlund, drehe mich um, drücke die Glastür auf und strebe wieder den Tassen zu, die sich zum Trocknen in einer Dampfwolke türmen. 
Die Espressomaschinen in Bahnhofcafés zeigen unübersehbar ihre Verwandtschaft mit den Lokomotiven, die Espressomaschinen von gestern und heute mit den Dampf- und E-Loks von gestern und heute. Ich mag hin- und herlaufen, mag mich drehen und wenden, soviel ich will: Ich sitze in einer Falle, in der zeitlosen Falle, die einem unweigerlich jeder Bahnhof stellt. Immer noch hängt ein feiner Kohlenstaub in der Luft, obwohl längst alle Strecken elektrifiziert worden sind, und ein Roman, der von Zügen und Bahnhöfen handelt, kann nicht umhin, diesen Rauchgeruch wiederzugeben. Schon mehrere Seiten hast du dich jetzt vorangelesen, es wäre mithin an der Zeit, daß dir klar gesagt wird, ob dieser Bahnhof, an dem ich ausgestiegen bin aus einem verspäteten Zug, ein Bahnhof von früher ist oder von heute; doch die Sätze bewegen sich weiter im ungewissen, im Grau, in einer Art Niemandsland der auf den kleinsten gemeinsamen Nenner verkürzten Erfahrung. Paß auf, das ist bestimmt ein Trick, um dich langsam einzufangen, dich in die Handlung hineinzuziehen, ohne daß du es merkst: eine Falle. (Calvino 1983, S. 16 f.)

\section{Annotationsrichtlinien}

Die nun folgenden Richtlinien dienen als praktische Arbeitsanleitung zur Annotation von Erzählebenen. Sie resultieren aus den explizierten Grundannahmen (s. Abschnitt 4). Bevor der Annotationsvorgang begonnen wird, sollte der zu annotierende literarische Text einmal komplett gelesen werden. Im Anschluss werden Erzählebenen wie folgt annotiert:

\subsection{Auszeichnung}

Alle Erzählebenen werden mit eckigen Klammern annotiert, wobei jede Ebene durch eine öffnende Klammer am Anfang und eine schließende Klammer am Ende gekennzeichnet wird. ${ }^{8}$

\subsection{Nummerierung}

Die unterschiedlichen Erzählebenen werden zunächst mit Nummern versehen (1, 2, 3 etc.). Abgesehen vom seltenen Sonderfall der Metalepse (s. Abschnitt 4.3.3) hat jeder Text mindestens eine Erzählebene, die mit der Nummer [1] gekennzeichnet wird. Sollte zusätzliche eine sequenzielle Reihe von Erzählebenen auftreten,

8 Die Annotation kann auch auf andere Weise erfolgen (z. B. mit unterschiedlichen Farben, die die Zugehörigkeit zu einer bestimmten Erzählebene markieren), je nachdem, welches Annotationswerkzeug verwendet wird. 
werden Buchstaben genutzt, um die nebeneinander angeordnete Ebenen zu benennen.

1. Die Nummer gibt den Grad der Erzählebene im Inklusionsschema an. Damit ist Erzählebene 2 eine Erzählung zweiter Stufe, die in eine übergeordnete Erzählung (Ebene 1) eingebettet ist. Es handelt sich bei der Erzählung zweiter Stufe um eine Binnenerzählung. So ist etwa das Märchen von ,Rip van Winkle“ in Max Frischs Roman Stiller eine Binnenerzählung, die in die Rahmenerzählung von Stillers Leben eingebettet ist.

2. Erzählungen, die sich auf derselben Erzählebene befinden, aber sequenziell angeordnet sind, werden mit Buchstaben ausgezeichnet (a, b, c). In Boccaccios berühmtem Novellenzyklus Il Decamerone finden sich einhundert sequenziell angeordnete Erzählungen, die alle auf der zweiten Ebene in die Rahmenerzählung eingebettet sind. Sie werden mit der Nummerierung 2a, 2b, 2c etc. versehen; in einem Extremfall wie dem von Boccaccios Zyklus müsste man sich mit doppelter und dreifacher Buchstabennennung aushelfen (... 2y, 2z, 2aa, 2ab).

\subsection{Verwendung der Klammern}

Die eckigen Klammern werden wie folgt platziert:

1. Sowohl die öffnende als auch die schließende Klammer sind mit der Nummer und ggf. einem Buchstaben gekennzeichnet: $\left[{ }_{1} \ldots\right]_{1}$

2. Die übergeordnete Erzählebene beginnt stets vor der eingebetteten Erzählung. Ebenso schließen die Klammern der übergeordneten Erzählung erst, wenn die Klammern der eingebetteten Erzählebenen geschlossen sind: $\left[\begin{array}{lll}{ }_{1} \ldots{ }_{2} & \ldots .\end{array}\right]_{2}$ .... $]_{1}$.

3. Bei sequenziellen Anordnungen schließen die Klammern der ersten sequenziell angeordneten Erzählung (z. B. 2a), bevor die der zweiten (z. B. 2b) sich öffnen: $\left.\left[\begin{array}{lllll}1 & \ldots & {[2 a} & \cdots\end{array}\right]_{2 a} \ldots\left[\begin{array}{lll}2 b & \ldots .\end{array}\right]_{2 b} \ldots\right]_{1}$.

4. Jegliche Interpunktion wird nicht vom vorangehenden Wort bzw. Satz getrennt.

(1) $\quad[]_{1} \ldots\left[\mathbf{L}_{2}, \text { Auf einem Marsch } 1792 \text { in der Rheinkampagne“, }\right]_{2}$ begann der Offizier, $\left[_{2}\right.$ „bemerkte ich, nach einem Gefecht, das wir mit dem Feinde gehabt hatten, einen Soldaten ..." $\left.]_{2}\right]_{1}$ (Kleist 1990, S. 376) 


\subsection{Eingebettete Erzählebenen und Herausgeberfiktion}

Jeder Text beginnt mit einer ersten Erzählebene, in die weitere Erzählebenen eingebettet sein können.

(2) [ 1 Zum kommenden Sonntag war ich von den Paulsenschen Eheleuten auf den Abend eingeladen, um ihnen ihren Hochzeitstag feiern zu helfen. Es war im Spätsommer, und da ich mich frühzeitig auf den Weg gemacht und die Hausfrau noch in der Küche zu wirtschaften hatte, so ging Paulsen mit mir in den Garten, wo wir uns zusammen unter der großen Linde auf die Bank setzten. Mir war das „Pole Poppenspäler“ wieder eingefallen, und es ging mir so im Kopf herum, daß ich kaum auf seine Reden Antwort gab; endlich, da er mich fast ein wenig ernst wegen meiner Zerstreuung zurecht gewiesen hatte, fragte ich ihn gradezu, was jener Beiname zu bedeuten habe.

Er wurde sehr zornig. „Wer hat dich das dumme Wort gelehrt?“ rief er, indem er von seinem Sitze aufsprang. Aber bevor ich noch zu antworten vermochte, saß er schon wieder neben mir. „Laß, laß!“ sagte er sich besinnend; „es bedeutet ja eigentlich das Beste, was das Leben mir gegeben hat. - Ich will es dir erzählen; wir haben wohl noch Zeit dazu. -

In diesem Haus und Garten bin ich aufgewachsen, meine braven Eltern wohnten hier, und hoffentlich wird einst mein Sohn hier wohnen! - Daß ich ein Knabe war, ist nun schon lange her; aber gewisse Dinge aus jener Zeit stehen noch, wie mit farbigem Stift gezeichnet, vor meinen Augen.

[ 2 Neben unserer Haustür stand damals eine kleine weiße Bank mit grünen Stäben in den Rück- und Seitenlehnen, von der man nach der einen Seite die lange Straße hinab bis an die Kirche, nach der andern aus der Stadt hinaus bis in die Felder sehen konnte. An Sommerabenden saßen meine Eltern hier, der Ruhe nach der Arbeit pflegend; in den Stunden vorher aber pflegte ich sie in Beschlag zu nehmen und hier in der freien Luft und unter erquickendem Ausblick nach Ost und West meine Schularbeiten anzufertigen. “ $\left.]_{2}\right]_{1}$ (Storm 1987, S. 166 f.)

Eine Erweiterung dieser Regel gibt es bei literarischen Texten, bei denen eine Herausgeberfiktion eine eigenständige Erzählebene evoziert. Sie wird als Rahmenerzählung auf Ebene 1 angegeben, auch wenn die Herausgeberfiktion erst am Ende der Erzählung sichtbar wird.

DECEMBER 6.

$I_{1}\left[_{2}\right.$ Wie mich die Gestalt verfolgt! Wachend und träumend füllt sie meine ganze Seele! Hier, wenn ich die Augen schließe, hier in meiner Stirne, wo die Sehkraft sich vereinigt, stehen ihre schwarzen Augen. Hier! ich kann dir es nicht ausdrücken. Mache ich meine Augen zu, so sind sie da; wie ein Meer, wie ein Abgrund ruhen sie vor mir, in mir, füllen die Sinne meiner Stirn.

Was ist der Mensch, der gepriesene Halbgott! Ermangeln ihm nicht eben da die Kräfte, wo er sie am nöthigsten braucht? Und wenn er in Freude sich aufschwingt, oder im Leiden versinkt, wird er nicht in beiden eben da aufgehalten, eben da zu dem stumpfen kalten Bewußtsein wieder zurückgebracht, da er sich in der Fülle des Unendlichen zu verlieren sehnte? $\left.]_{2}\right]_{1}$ 
Der Herausgeber an den Leser.

[ 1 Wie sehr wünscht' ich, daß uns von den letzten merkwürdigen Tagen unsers Freundes so viel eigenhändige Zeugnisse übrig geblieben wären, daß ich nicht nöthig hätte, die Folge seiner hinterlassenen Briefe durch Erzählung zu unterbrechen. $]_{1}$ (Goethe 1899, S. 139-141)

\subsection{Paratexte}

Wie schon in Beispiel (3) ersichtlich, werden Paratexte (vgl. Genette 1989) wie Buchtitel, Vorworte, Kapitelüberschriften und Gattungsangaben nicht annotiert. Wenn sich die Erzählebene nicht ändert, werden die Klammern am Ende des vorangehenden Kapitels geschlossen und erst nach der Überschrift wieder geöffnet.

(4) [ ${ }_{1}$ By reason of these things, then, the whaling voyage was welcome; the great floodgates of the wonder-world swung open, and in the wild conceits that swayed me to my purpose, two and two there floated into my inmost soul, endless processions of the whale, and, midmost of them all, one grand hooded phantom, like a snow hill in the air. $]_{1}$

CHAPTER 2

The Carpet-Bag

[ ${ }_{1}$ I stuffed a shirt or two into my old carpet-bag, tucked it under my arm, and started for Cape Horn and the Pacific. Quitting the good city of old Manhatto, I duly arrived in New Bedford. It was on a Saturday night in December. Much was I disappointed upon learning that the little packet for Nantucket had already sailed, and that no way of reaching that place would offer, till the following Monday. $]_{1}$ (Melville 2002, S. 22 f.)

\subsection{Syntaktisch eingebundene Überschriften}

Überschriften, die jedoch semantisch und syntaktisch zur aktuellen Erzählebene gehören, bilden hierzu eine Ausnahme. Sie werden zur zugehörigen Erzählebene geordnet.

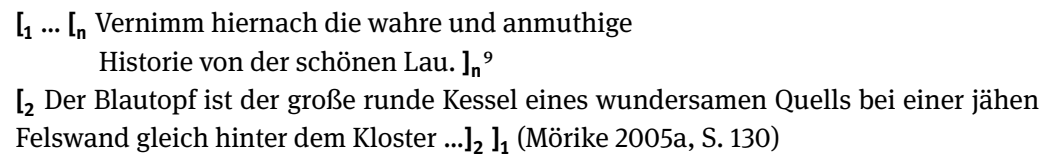

9 Die Abkürzung n steht für ,nicht erzählend‘ und wird in Abschnitt 5.8 erklärt. 


\subsection{Unterbrechung von Erzählebenen}

Erzählebenen können durch andere Erzählebenen unterbrochen werden. So können im Erzählakt eines sekundären Erzählers etwa Aussagen vorkommen, die auf der primären Erzählebene stattfinden. Zwei Fälle solcher Unterbrechungen sind möglich:

1. eingefügte verba dicendi, die auf die spezifische Erzählsituation verweisen und damit noch zur übergeordneten Erzählebene gehören, und

2. eingeschobene Sprechakte, die nicht Teil der Erzählung sind, sondern sich etwa an die Figuren auf der übergeordneten Erzählebene richten.

In solchen Fällen wird die eingeschobene Erzählebene geschlossen, wenn der Einschub beginnt, und wieder geöffnet, wenn er endet. In Beispiel (6) werden drei sequenziell angeordnete Geschichten erzählt (2a, 2b, 2c), wobei hier nur die dritte zu sehen ist. Diese wird durch Einschübe auf der ersten Erzählebene unterbrochen. Dabei handelt es sich einerseits um einen Einschub mit verbum dicendi (,ergänzte der Offizier“), andererseits um eine Ansprache des Adressatenkreises („meine Herren“). Beide Formen der Unterbrechung können auch gemeinsam auftreten, müssen in diesem Fall aber nicht separat annotiert werden (Beispiel (7): ,,Nun also', sagte er."). Einschübe dieser Art können im Übrigen auch nur aus einem Wort bestehen, ebenso wie sie mehrere Sätze umfassen können.

(6) [ ${ }_{1}$ Der Landedelmann meinte, daß er die Geschichten, die seinen Satz belegen sollten, gut zu wählen wüßte.

[2c „Die dritte Geschichte, “ $]_{2 c}$ fuhr der Offizier fort, [ $2 \mathrm{c}$,trug sich zu, im Freiheitskriege der Niederländer, bei der Belagerung von Antwerpen durch den Herzog von Parma. Der Herzog hatte die Schelde, vermittelst einer Schiffsbrücke, gesperrt, und die Antwerpner arbeiteten ihrerseits, unter Anleitung eines geschickten Italieners, daran, dieselbe durch Brandner, die sie gegen die Brücke losließen, in die Luft zu sprengen. In dem Augenblick, " $]_{2 c}$ meine Herren, ${ }_{2 c}$ da die Fahrzeuge die Schelde herab, gegen die Brücke anschwimmen, steht, das merken sie wohl, ein Fahnenjunker, auf dem linken Ufer der Schelde, dicht neben dem Herzog von Parma; jetzt, $]_{2 c}$ verstehen sie, $[2 c$ jetzt geschieht die Explosion; und der Junker, Haut und Haar, samt Fahne und Gepäck, und ohne daß ihm das Mindeste auf dieser Reise zugestoßen, steht auf dem Rechten. $]_{2 c}$ Und die Schelde ist hier, wie Sie wissen werden, einen kleinen Kanonenschuß breit. „Haben Sie verstanden?“

Himmel, Tod und Teufel! rief der Landedelmann .... $]_{1}$ (Kleist 1990, S. 378 f.)

(7) $\left.\quad I_{1}\right]_{2}$...Der Alte sah mich mit einem verständnisvollen Lächeln an: „Nun also!“ sagte er. $\left[_{3}\right.$,In der Mitte des vorigen Jahrhunderts, oder vielmehr, um genauer zu bestimmen, vor und nach derselben, gab es hier einen Deichgrafen, der von Deich- und Sielsachen 
mehr verstand, als Bauern und Hofbesitzer sonst zu verstehen pflegen; aber es reichte doch wohl kaum; denn was die studierten Fachleute darüber niedergeschrieben, davon hatte er wenig gelesen; sein Wissen hatte er sich, wenn auch von Kindesbeinen an, nur selber ausgesonnen. “ $]_{3}$ Ihr hörtet wohl schon, Herr, die Friesen rechnen gut, und habet auch wohl schon über unseren Hans Mommsen von Fahretoft reden hören, der ein Bauer war und doch Boussolen und Seeuhren, Teleskopen und Orgeln machen konnte. Nun, $\left[_{3}\right.$ ein Stück von solch einem Manne war auch der Vater des nachherigen Deichgrafen gewesen; freilich wohl nur ein kleines .... $]_{3}$.... $\left.]_{2}\right]_{1}$ (Storm 1988, S. 639)

\subsection{Nicht-erzählende Passagen}

Es gibt Situationen, in denen der Erzähler seine Geschichte unterbricht, um diese $z u$ kommentieren - allerdings ohne eine Figur einer anderen Erzählebene in der fiktionalen Welt anzusprechen (s. Abschnitt 3.4). Solche Formen von Aphorismen, Motti, Kommentare, Urteile, Gedanken und - auf primärer Erzählebene Formen der Ansprache an den fiktionalen Adressaten (vgl. Schmid 2014, S. 7) werden als Teil der aktuellen Erzählebene verstanden und nicht als eigenständige Erzählebene betrachtet. Da es in einigen Fällen - etwa um das Urteil des Erzählers mit der Handlung zu vergleichen - aufschlussreich sein kann, annotieren wir diese Ausdrücke als ,nicht erzählend'. Hierfür verwenden wir eckige Klammern und den Buchstaben n, womit angedeutet wird, dass solche Textstellen weder eine eigenständige Erzählebene bilden noch zur übergeordneten Erzählebene gehören (s. Abschnitt 5.7). Mit öffnenden Klammern wird der Beginn und mit schließenden Klammern das Ende des Ausdrucks markiert. Die Beispiele (8), (9) und (10) enthalten Elemente, die einen fiktiven Empfänger ansprechen und daher als nicht erzählter Teil betrachtet werden können.

(8) [ ${ }_{1}$ That puzzled the Leopard and the Ethiopian, but they set off to look for the aboriginal Flora, and presently, after ever so many days, they saw a great, high, tall forest full of tree trunks all 'sclusively speckled and sprottled and spottled, dotted and splashed and slashed and hatched and cross-hatched with shadows. [n (Say that quickly aloud, and you will see how very shadowy the forest must have been.) $]_{n}$.... $]_{1}$ (Kipling 1968, S. 47)

(9) [ ${ }_{1}$ Das war die erste lange Trennung, fast auf zwölf Stunden. $\left[_{n} \text { Arme Eff!! }\right]_{n}$ Wie sollte sie den Abend verbringen? Früh zu Bett, das war gefährlich, dann wachte sie auf und konnte nicht wieder einschlafen und horchte auf alles. Nein, erst recht müde werden und dann ein fester Schlaf, das war das Beste. Sie schrieb einen Brief an die Mama und ging dann zu Frau Kruse, deren gemütskranker Zustand - sie hatte das schwarze Huhn 
oft bis in die Nacht hinein auf ihrem Schoß - ihr Teilnahme einflößte. $]_{1}$ (Fontane 1998, S. 79$)^{10}$

(10) $\quad\left[{ }_{1}\right.$ In the days when everybody started fair, $[\mathbf{n} \text { Best Beloved }]_{n}$, the Leopard lived in a place called the High Veldt. $\left[_{n} \text {, Member }\right]_{n}$ it wasn't the Low Veldt, or the Bush Veldt, or the Sour Veldt, but ... $]_{1}$ (Kipling 1968, S. 41)

\section{Primärliteratur}

Abbé Prévost (2008). Manon Lescaut. Übers. von Walter Widmer. Zürich: Diogenes.

Calvino, Italo (1983). Wenn ein Reisender in einer Winternacht. Wien/München: Carl Hanser Verlag.

Fontane, Theodor (1998). Effi Briest. Hrsg. von Christine Hehle. Bd. 8. Große Brandenburger Ausgabe. Berlin: Aufbau-Verlag.

Frisch, Max (1954). Stiller. Frankfurt am Main: Suhrkamp.

Goethe, Johann Wolfgang (1899). Die Leiden des jungen Werther. i. A.d. Großherzogin Sophie von Sachsen. Weimar: Hermann Böhlaus Nachfolger, S. 1-192.

Joyce, James (1967). „Eveline“. In: Dubliners. Hrsg. von Robert Scholes. Bungay/Suffolk: The Chaucer Press, S. 37-44.

Kipling, Rudyard (1968). Just So Stories. For Little Children. London/Toronto/Melbourne: Macmillan, S. 41-59.

Kleist, Heinrich von (1990). Unwahrscheinliche Wahrhaftigkeiten. Hrsg. von Klaus Müller-Salget. Bd. 3: Erzählungen, Anekdoten, Gedichte, Schriften. Sämtliche Werke und Briefe in vier Bänden. Frankfurt am Main: Deutscher Klassiker Verlag, S. 376-379.

Melville, Herman (2002). Moby-Dick. Hrsg. von Herhel Parker und Harrison Hayford. New York/London: Norton \& Company.

Mörike, Eduard (2005a). Das Stuttgarter Hutzelmännlein. Hrsg. von Matthias Mayer. Bd. 6.1: Erzählungen, Erster Teil. Werke und Briefe. Stuttgart: Klett-Cotta, S. 119-168.

Mörike, Eduard (2005b). Lucie Gelmeroth. Hrsg. von Matthias Mayer. Bd. 6.1: Erzählungen, Erster Teil. Werke und Briefe. Stuttgart: Klett-Cotta, S. 11-29.

Storm, Theodor (1987). Pole Poppenspäler. Hrsg. von Karl Ernst Laage und Dieter Lohmeier. Bd. 2: Novellen 1867-1880. Sämtliche Werke in vier Bänden. Frankfurt am Main: Deutscher Klassiker Verlag, S. 164-221.

Storm, Theodor (1988). Der Schimmelreiter. Hrsg. von Karl Ernst Laage. Bd. 3: Novellen 18811888. Sämtliche Werke in vier Bänden. Frankfurt am Main: Deutscher Klassiker Verlag, S. 634-756.

Tschechow, Anton Pawlowitsch (1968). „Tschechows Erzählungen“. In: Das Gewinnlos. Übers. von Leo Borchard und L. Flachs-Fokschneanu. Harenberg, S. 63-67.

10 Erläuterung zu Beispiel 9: Die Sätze „Früh zu Bett, das war gefährlich, dann wachte sie auf und konnte nicht wieder einschlafen und horchte auf alles. Nein, erst recht müde werden und dann ein fester Schlaf, das war das Beste“ sind erlebte Rede und daher kein wertender Kommentar des Erzählers wie „Arme Effi!“. 


\section{Sekundärliteratur}

Abbot, H. Porter (2014). „Narrativity“. In: Handbook of Narratology. Hrsg. von John Pier und Wolf Schmid. Berlin: De Gruyter, S. 587-607.

Coste, Didier und John Pier (2009). „Narrative Levels“. In: Handbook of Narratology. Hrsg. von John Pier, Wolf Schmid und Jörg Schönert. Berlin: De Gruyter, S. 295-309.

Fludernik, Monika (2006). Einführung in die Erzähltheorie. Darmstadt: WBG.

Genette, Gérard (1989). Paratexte. Das Buch vom Beiwerk des Buches. Frankfurt am Main/New York: Campus Verlag.

Genette, Gérard (1994). Die Erzählung. Übers. von Andreas Knop. München: Fink. Igl, Natalie (2018). „Erzähler und Erzählerstimme“. In: Grundthemen der Literaturwissenschaft: Erzählen. Hrsg. von Martin Huber und Wolf Schmid. Berlin: De Gruyter, S. 127-149.

Jahn, Manfred (2017). Narratology. A Guide to the Theory of Narrative. Köln: Universität Köln. URL: http://www.uni-koeln.de/\%7Eame02/pppn.htm (besucht am 1. Juni 2020).

Ketschik, Nora, Benjamin Krautter, Sandra Murr und Yvonne Zimmermann (2019). „Annotation Guideline No. 4: Annotating Narrative Levels in Literature“. In: Cultural Analytics: A Shared Task for the Digital Humanities: Annotating Narrative Levels. DoI: 10.22148/16.055.

Klausnitzer, Ralf (2013). „Institutionalisierung und Modernisierung der Literaturwissenschaft seit dem 19. Jahrhundert“. In: Handbuch Literaturwissenschaft. Hrsg. von Thomas Anz. Bd. 3. Metzler, S. 70-147.

Lahn, Silke und Jan-Christoph Meister (2013). Einführung in die Erzähltextanalyse. 2. Aufl. Stuttgart: Metzler.

Margolin, Uri (2014). „Narrator“. In: Handbook of Narratology. Hrsg. von Peter Hühn, Jan Christoph Meister, John Pier, Wolf Schmid und Jörg Schönert. Hamburg: Hamburg University Press. URL: https://www.lhn.uni-hamburg.de/node/44/revisions/296/view.html (besucht am 1. Juni 2020).

Martínez, Matías und Michael Scheffel (2009). Einführung in die Erzähltheorie. München: C.H.Beck.

Meister, Jan Christoph (2014). „Narratology“. In: Handbook of Narratology. Hrsg. von Peter Hühn, Jan Christoph Meister, John Pier, Wolf Schmid und Jörg Schönert. Berlin: De Gruyter, S. $623-645$.

Pier, John (2014). „Narrative Levels“. In: Hrsg. von Peter Hühn, Jan Christoph Meister, John Pier, Wolf Schmid und Jörg Schönert. Berlin: De Gruyter, S. 547-563.

Pier, John (2016). „Metalepsis“. In: The Living Handbook of Narratology. Hrsg. von Peter Hühn, John Pier, Wolf Schmid und Jörg Schönert. Hamburg: Hamburg University Press. URL: https: //www.lhn.uni-hamburg.de/node/51.html (besucht am 1. Juni 2020).

Reiter, Nils (2020). „Anleitung zur Erstellung von Annotationsrichtlinien“. In: Reflektierte Algorithmische Textanalyse. Hrsg. von Nils Reiter, Axel Pichler und Jonas Kuhn. Berlin: De Gruyter, S. 193-201.

Ryan, Marie-Laure (1991). Possible Worlds, Artificial Intelligence and Narrative Theory. Bloomington, Indiana: Indiana University Press.

Ryan, Marie-Laure (2001). „The Narratorial Functions. Breaking Down a Theoretical Primitive“. In: Contemporary Narratology 9.2, S. 146-152.

Schmid, Wolf (2003). „Narrativity and Eventfullness“. In: What is Narratology. Questions and Answers Regarding the Status of a Theory. Hrsg. von Tom Kindt und Hans-Harald Müller. Berlin: De Gruyter, S. 17-35. 
Schmid, Wolf (2014). Elemente der Narratologie. 3. Aufl. Berlin: De Gruyter.

Schmid, Wolf (2018). „Ereignis“. In: Grundthemen der Literaturwissenschaft. Hrsg. von Martin Huber und Wolf Schmid. Berlin: De Gruyter, S. 312-333. 